\title{
ACEPTABILIDAD DEL ENSILAJE DE LA PARTE AÉREA DE LA YUCA ADICIONADO CON DIFERENTES TIPOS Y CANTIDADES DE ADITIVOS EN BOVINOS
}

\section{ACCEPTABILITY SILAGE AIR PART OF YUCCA ADDED WITH DIFFERENT TYPES AND QUANTITIES OF ADDITIVES IN CATTLE}

\author{
PÉREZ, P. JORGE,,$^{\star}{ }^{2}$ Zoot, PATIÑO, P. RENÉ, ${ }^{2}$ Dr, ROMERO, P. ALBERTO,${ }^{3}$ Zoot, \\ RIVERO, E. SANDRA, ${ }^{4}$ Dr, SALCEDO, C. ERICA, ${ }^{4}$ Zoot, SUAREZ, P. EMIRO,${ }^{4}$ Zoot. \\ ${ }^{1}$ Universidad Federal De Lavras, Av. Doutor Sylvio Menicucci, 1001 - Kennedy, Lavras - MG, 37200-000, Brasil. \\ 2 Universidad de Sucre, Facultad de Ciencias Agropecuarias, Sincelejo - Colombia. \\ ${ }^{3}$ Universidad de Sucre, Sincelejo - Colombia \\ ${ }^{4}$ CORPOICA - Turipana, Cerete - Córdoba.
}

Palabras Clave:

Consumo,

Salvado de arroz,

Tusa de maíz.

Key words:

Intake,

Rice bran,

Corncob.

\section{Resumen}

El objetivo de esta investigación fue evaluar la influencia de la adición de diferentes tipos y proporciones de subproductos y aditivos al ensilaje de la parte aérea de la yuca sobre su aceptabilidad a diferentes tiempos de ensilado, asociando las variables organolépticas, de composición química y aceptabilidad del ensilaje. Se utilizó un diseño completamente al azar con siete tipos o proporciones de aditivos y cuatro tiempos de ensilado. Los tratamientos fueron: como testigo, la parte aérea de la yuca adicionada con $2 \%$ de melaza, adición común en todos los tratamientos; Sil-All囚 (inoculante bacteriano); salvado de arroz ( 15 y $30 \%$ de inclusión); tusa de maíz molida (15 y $30 \%$ de inclusión) y azúcar (3\% de inclusión). Los ensilajes adicionados con tusa fueron mayormente aceptados por los animales, reflejado esto en mayor número y tiempo de visitas y un mayor consumo. Los ensilajes que incluyeron salvado fueron los menos aceptados. La edad de abertura del silo no afectó $(P>0.05)$ las variables en estudio. Se confirmó la preferencia de los animales por el material ensilado en comparación al material no ensilado. La aceptabilidad de los materiales ensilados se relacionó con algunas variables de calidad, como $\mathrm{pH}$, FDA, hemicelulosa, grasa y la DISMS.

\footnotetext{
Abstract

The objective of this research was to evaluate the influence of the addition of different types and proportions of byproducts and silage additives to the aerial part of cassava on their acceptability silage at different times, involving the sensory variables, chemical composition and acceptability silage. Experimental design was completely randomized with seven types or proportions of additives and four times silage. Treatments were as a witness, the aerial part of cassava supplemented with $2 \%$ molasses, common addition in all treatments; Sil-AlI囚 (bacterial inoculant); rice bran (15 and $30 \%$ inclusive); ground corn cobs (15 and $30 \%$ inclusion) and sugar (3\% inclusion). The silages added with tusa were largely accepted by the animals, reflected this in greater number and timing of visits and increased consumption. The silages that included bran were the least accepted. The opening of the silo age did not affect $(P>0.05)$ the study variables. The preference of the animals for the ensiled material compared to silage material not confirmed. The acceptability of the ensiled material was related to some quality variables such as $\mathrm{pH}$, ADF, hemicellulose, fat and DISMS.
} 


\section{Introducción}

La ganadería es una actividad importante en el departamento de Sucre y en toda la región Caribe, tanto cultural como económicamente, pero debido a la forma como se distribuye la lluvia durante el año, la producción y oferta forrajera se ven afectadas notoriamente, ya sea por escases en la época seca o por las inundaciones en la época de lluvias (AGUILERA, 2005; CUADRADO et al., 2003; SÁNCHEZ, 2005). Adecuar el inventario de animales según la oferta de la época, almacenar forrajes conservados en forma de silos o henos, o en la forma de pastoreo diferido, implementar algún tipo de riego, ofrecer suplementos alimenticios, como granos, concentrados o subproductos de la agroindustria, entre otros, son algunas de las estrategias utilizadas por los ganaderos para enfrentar este problema.

La preservación de forrajes empleando un ambiente ácido (ensilaje), es un método práctico y económico para utilizar forrajes eficientemente, que permite conservar sus características nutritivas en el tiempo, para ser utilizado en épocas de escases (GARCÉs et al., 2004). La yuca (Manihot esculenta) gracias a su alta producción, contenido nutricional y adaptación a zonas tropicales es comúnmente utilizado para alimentar animales, principalmente bovinos (ROCERO, 2002). Sin embargo, cuando este material está en su punto óptimo de calidad y de producción de materia seca presenta una elevada proporción de humedad, que usualmente supera el $75 \%$, lo que representa una limitante para obtener ensilajes de buena calidad, ocasionando aumento en los costos de producción y variaciones en la respuesta esperada (FERNANDEZ, 1999).

Se ha establecido que los factores que influyen y condicionan el consumo voluntario de alimentos en los rumiantes están clasificados como factores inherentes al animal, donde el sistema nervioso central juega un papel indispensable para el control de la ingestión de alimentos por el animal, la cual está controlada por mecanismos fisiológicos que llevan al animal a iniciar y a finalizar el consumo en un momento dado, factores inherentes a la dieta, los cuales están asociados con atributos propios del alimento como composición nutricional, palatabilidad, formas físicas y a su vez la capacidad del tracto digestivo del animal, por ultimo factores ambientales lo cuales influyen directa e indirectamente en los anteriores, pudiendo citar, temperatura, humedad, radiación, velocidad del viendo, entre otros, los cuales permiten o no un ambiente adecuado para expresar el potencial productivo del animal (ARAUJO, 2005; BARAHONA y SÁNCHEZ, 2005; CARVALHO et al., 2011; FERREIRA, 2005; MEDRANO, 1991). Aspectos relacionados con el comportamiento ingestivo animal están siendo utilizados para evaluar planes nutricionales y suplementos, a fin de predecir la respuesta animal frente a una variación en la oferta de alimento. Cabe resaltar la importancia de este método de evaluación, que en los últimos años ha sido utiliza por muchos autores debido a la amplitud de la información que puede ser obtenida a partir de estos estudios (MARTINEZ et al., 2002; ORTEGA et al., 2009; PATIÑO et al., 2008).

En este contexto, se evaluó la influencia de la adición de diferentes tipos y proporciones de subproductos y aditivos al ensilaje de la parte aérea de la yuca sobre su aceptabilidad a diferentes tiempos de ensilado, asociando las variables organolépticas, de composición química y aceptabilidad del ensilaje.

\section{Materiales y métodos}

La investigación fue realizada en las instalaciones del centro de investigaciones Turipaná CORPOICA, coordenadas 8.86495666412, - 75.7658514593, $\mathrm{Km} 13$ vía Montería - Cereté, Córdoba, con temperatura promedio de $28{ }^{\circ} \mathrm{C}$, humedad relativa de $97 \%$ y precipitación promedio anual de $1200 \mathrm{~mm}$, presentándose dos épocas durante el año (sequía y lluvias). El material para la elaboración de los ensilajes se obtuvo de la variedad de yuca CORPOICA.SM 208134 , considerada como forrajera. La parte aérea de la planta fue cortada manualmente a $40 \mathrm{~cm}$ del suelo y luego picada con tamaño de partícula $1,5 \mathrm{~cm}$. Para el empaque fueron utilizados micro-silos, fabricados en plástico de color negro de calibre 8 , con $115 \mathrm{~cm}$ de alto por $62 \mathrm{~cm}$ de ancho y una capacidad de $15 \mathrm{~kg}$ de material. Fueron considerados los días $0,20,40$ y 60 de ensilado para realizar las respectivas observaciones de comportamiento ingestivo.

Los tratamientos utilizados en este trabajo fueron: Tusa $15 \%$ (Ensilaje de la parte aérea de la yuca adicionado con $15 \%$ de tusa de maíz), Tusa 30\% (Ensilaje de la parte aérea de la yuca adicionado con $30 \%$ de tusa de maíz), Salvado 15\% (Ensilaje de la parte aérea de la yuca adicionado con $15 \%$ de salvado de arroz), Salvado $30 \%$ (Ensilaje de la parte aérea de la yuca adicionado con $30 \%$ de Salvado de arroz), Sil-all $®$ (Ensilaje de la parte aérea de la yuca adicionado con Sil-all囚), Testigo Hy (Ensilaje de la parte aérea de la yuca), Azúcar (Ensilaje de la parte aérea de la yuca adicionado con $4 \%$ de azúcar). Las observaciones de comportamiento ingestivo se llevaron a cabo en tres corrales de $6 \mathrm{~m}^{2}$ cada uno. Dentro de cada corral se colocaron siete comederos de madera $(45 \times 60 \times$ $40 \mathrm{~cm}$ ) y un bebedero con capacidad de cuatro litros, con regulación constante del volumen de agua. Como material de cama se utilizó cascarilla de arroz. Para las observaciones fueron utilizadas tres vacas del mismo grupo racial, de similar peso y condición corporal, edad y fase fisiológica (secas), ubicadas en cada corral. A cada 
una se le ofrecieron los siete tratamientos, los cuales se distribuyeron al azar en los comederos del corral. Se realizaron un total de siete observaciones, dos en los días 20,40 y 60 y una en el día cero, que correspondió al material fresco. Se observó a los animales durante 12 horas, desde las 6:00 hasta las 18:00 horas. Se registró las veces en que el animal llego a cada material y además el tiempo que permaneció consumiendo en cada tratamiento. El consumo diario fue calculado a través de la diferencia entre la cantidad ofrecida y la sobrante. Además se hicieron los respectivos análisis de las variables de composición nutricional: materia seca, proteína bruta, fibra detergente neutro, fibra detergente acida, extracto etéreo, cenizas, digestibilidad in situ de la MS y $\mathrm{pH}$, llevados a cabo en un estudio paralelo a este, el cual tuvo como objetivo estudiar los mismos tratamientos y su efecto sobre la composición nutricional de los ensilajes, esto con el fin de asociar dichas variables con la aceptabilidad de los ensilajes por parte del animal.

El diseño estadístico utilizado fue bloques al azar en arreglo factorial (siete tipos o proporciones de aditivos y cuatro tiempos de ensilado); el factor día de observación fue considerado como medida repetida en el tiempo. De esta manera, el modelo general incluyó los efectos del tratamiento (seis grados de libertad), edad de ensilado (3 grados de libertad), la interacción entre tratamiento y periodo, y los respectivos errores. Se realizó análisis de varianza y las medias se compararon mediante la prueba de Tukey al $5 \%$ de significancia. Los datos referentes al consumo de MS y tiempo de consumo de cada material fueron analizados usando la versión 2011 de InfoStat. La estimación de los modelos lineales y mixtos fue a través de los procedimientos GLS y LME de la librería NLME. El valor de probabilidad establecido como significativa fue menor a 0,05. Niveles de significancia 0,05 y 0,1 se consideraron como tendencia. Para la frecuencia de visitas se realizó un análisis de frecuencias. Para estudiar el grado de asociación entre las diferentes variables se usó el análisis de componentes principales, usando el mismo paquete estadístico. Para estudiar la relación entre las variables de aceptabilidad y composición nutricional de los diferentes materiales se aplicó un análisis de regresión de Pearson (DI RENZO, 2011).

\section{Resultados}

Los ensilados más visitados por los animales fueron los que incluyeron tusa, en ambas proporciones (15\% y $30 \%$ ). El ensilado con $15 \%$ de inclusión de tusa y con apertura en el dia 20 presentó el mayor número de visitas por día $(7,67)$, mientras que, para el adicionado con $30 \%$ de tusa, el mayor número de visitas correspondió al material abierto el dia 60 (11 Visitas/ día). Los tratamientos menos visitados por los animales fueron los que incluyeron salvado, manifestando mayor interés en el día 0 (material fresco). Los demás tratamientos presentaron números de visita intermedios pero mayores en el día 20 de ensilado el material. Las anteriores tendencias se pueden observar en la Tabla 1.

Tabla 1. Número de visitas diarias para los tratamientos de ensilaje de la parte aérea de la yuca adicionado con diferentes aditivos y tiempos de conservación por parte de vacunos.

\begin{tabular}{ccccc}
\hline \multirow{2}{*}{ Tratamientos } & \multicolumn{4}{c}{ Medias $\mathbf{N}^{\circ}$ visitas } \\
\cline { 2 - 5 } & Día 0 & Día 20 & Día 40 & Día 60 \\
\hline Tusa 15 & 3,33 & 7,67 & 5,5 & 4,67 \\
Tusa 30 & 3,67 & 4,83 & 7,67 & 11 \\
Salvado 15 & 3,67 & 0,83 & 1,67 & 2,5 \\
Salvado 30 & 2,33 & 1,33 & 1,5 & 1,17 \\
Sil-All囚 & 2 & 5,67 & 1,33 & 0,67 \\
Testigo Hy & 4 & 5,67 & 3,17 & 2,67 \\
Azúcar & 2 & 4 & 2,33 & 4,67 \\
\hline Total & $\mathbf{2 1}$ & $\mathbf{3 0}$ & $\mathbf{2 3 , 1 7}$ & $\mathbf{2 7 , 3 5}$ \\
\hline
\end{tabular}

Tusa 15\% (Ensilaje adicionado con 15\% de tusa de maíz), Tusa 30\% (Ensilaje adicionado con $30 \%$ de tusa de maíz), Salvado $15 \%$ (Ensilaje adicionado con $15 \%$ de salvado de arroz), Salvado 30\% (Ensilaje adicionado con 30\% de Salvado de arroz), Sil-all $($ Ensilaje adicionado con Sil-all@), Testigo Hy (Ensilaje de la parte aérea de la yuca), Azúcar (Ensilaje adicionado con $4 \%$ de azúcar).

Los tiempos de consumo o tiempos de presencia en los comederos variaron de acuerdo al tratamiento y al día de ensilado el material, indistintamente de esto, el mayor $(0,05 \%)$ tiempo de consumo se presentaban en las horas de la media mañana y en las horas frescas de la tarde. Tusa 30 tuvo diferencias significativas $(0,05 \%)$ en el día 60 respecto a los días 20 y 40 y a la vez estos con el día 0 . Tratamientos con salvado no se encontraron diferencias significativas (Figura 1).

Indistintamente del tratamiento, se observó que los menores consumos se obtuvieron en los días 0 (material no ensilado), inclusive con diferencias estadísticamente $(P<0,05)$ para los tratamientos Tusa 15 , Tusa 30 , Sil$A \| \circledast$ y azúcar, lo que evidencia una clara preferencia

\section{TIEMPO DE CONSUMO}

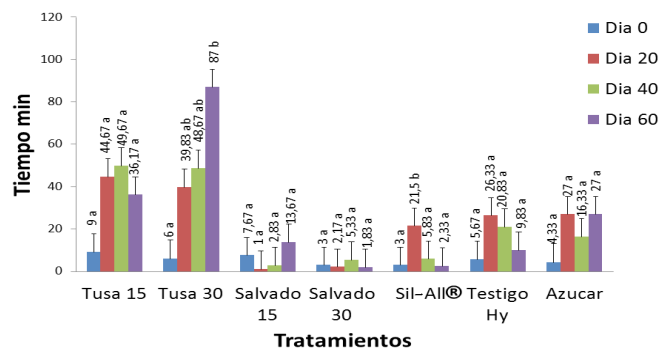

Figura 1.Tiempo de consumo para los tratamientos de ensilaje de la parte aérea de la yuca adicionado con diferentes aditivos y tiempos de conservación por parte de vacunos. 
de los animales por el material ensilado (Figura 2). En relación a tratamientos, aquellos que incluyeron tusa fueron mayormente consumidos $(P<0,05)$ por los animales, mostrando preferencias por el día 40. Los tratamientos con salvado presentaron los menores $(\mathrm{P}<0,05)$ consumos.

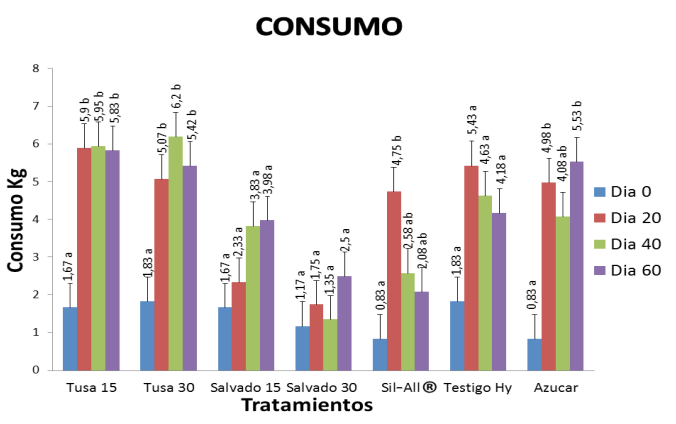

Figura 2. Consumo de ensilaje de la parte aérea de la yuca adicionado con diferentes aditivos y tiempos de conservación por parte de vacunos.

Tusa 15\% (Ensilaje adicionado con 15\% de tusa de maíz), Tusa $30 \%$ (Ensilaje adicionado con $30 \%$ de tusa de maíz), Salvado 15\% (Ensilaje adicionado con $15 \%$ de salvado de arroz), Salvado 30\% (Ensilaje adicionado con 30\% de Salvado de arroz), Sil-all $($ (Ensilaje adicionado con Sil-all $囚$ ), Testigo Hy (Ensilaje de la parte aérea de la yuca), Azúcar (Ensilaje adicionado con $4 \%$ de azúcar). Medias con letras iguales no difieren significativamente $(P=<0,05)$

Al correlacionar las variables de calidad de los ensilajes adicionados con diferentes aditivos, se observó que variables como $\mathrm{pH}(\mathrm{r}-0,63 \mathrm{P}<0,001)$, Hemicelulosa $(r-0,43 P<0,001)$, Extracto etéreo $(r-0,32 P 0,01)$ y Digestibilidad de la materia seca $(r-0,28$ P0,02) presentaron una correlación negativa con el consumo y solo el parámetro FDA ( $0,66 \mathrm{P}<0,001)$ presento correlación positiva. Las demás variables no se consideran significativas (Tabla 2).

Tabla 2. Correlaciones significativas de variables de calidad del ensilaje de la parte aérea de la yuca con el consumo.

\begin{tabular}{ccc}
\hline \multirow{2}{*}{ Variables de calidad } & \multicolumn{2}{c}{ Consumo } \\
\cline { 2 - 3 } & $\mathbf{R}$ & $\mathbf{p}$ \\
\hline pH & $\mathrm{r}=-0,63$ & $\mathrm{P}<0.001$ \\
FDA & $\mathrm{r}=0,66$ & $\mathrm{P}<0.001$ \\
HEMIC & $\mathrm{r}=-0,43$ & $\mathrm{P}<0.001$ \\
EE & $\mathrm{r}=-0,32$ & $\mathrm{P} 0.01$ \\
DISMS & $\mathrm{r}=-0,28$ & $\mathrm{P} 0,02$ \\
\hline
\end{tabular}

Donde FDA: fibra detergente acida, HEMIC: hemicelulosa, EE: extracto etéreo, DSMS: digestibilidad de la materia seca, $r=$ el valor de cada correlación, $p=e$ el valor de significancia de la correlación.

\section{Discusión}

Al incluir aditivos al ensilaje de la parte aérea de la yuca se mejoró notablemente el contenido de materia seca y con ella las características propias del ensilaje, cada aditivo en cada proporción modificó las características del ensilaje a lo largo del tiempo de ensilado, sin embargo, al hacer las pruebas de comportamiento se identificó que no siempre lo más nutritivo es lo más aceptado por el animal, y que la respuesta del animal ante la oferta de una dieta está correlacionada a una serie de características nutricionales y no nutricionales que pueden alterar el potencial de un tipo de alimento ser consumido. Esto fue estudiado por varios autores, dentro de los cuales ARAUJO (2005) destaca que una serie de factores propios del animal (hormonas, capacidad ruminal, estado fisiológico), el alimento (composición nutricional, tamaño de partícula, palatabilidad) y el ambiente (temperatura, humedad relativa) regulan el consumo voluntario de los rumiantes.

De manera general, en este estudio, los animales prefirieron el material ensilado, esto lo muestran las diferencias significativas en relación al día 0 , indicando que los parámetros sensoriales como sabor y olor propios del ensilaje ejercen un estímulo sobre los animales que conlleva a la preferencia de este en relación al material fresco. Otro aspecto que pudo influir en esta tendencia es la misma presentación del material, los materiales del día 0 se apreciaban mucho más ásperos al tacto, secos y con presencia de harinas en el caso del salvado. ALLISOM (1985) estableció que el consumo de 5 pastos, de 11 que fueron estudiados, fue estimulado por su sabor, color y textura, lo que significa que existió estimulación sensorial. De la misma forma FERNANDEZ DE SOUZA et al (2011) demostró que en el caso de ensilajes, una inadecuada fermentación producto de un mal manejo del silo o por lo general por un inadecuado contenido de MS provocan un comportamiento anormal del $\mathrm{pH}$ que acarrea la aparición de microorganismos indeseables que contaminan el material provocando olores y sabores que limitan su consumo.

Al ofrecer ensilaje de la parte aérea de la yuca adicionado con diferentes tipos y cantidades de aditivos a los animales se evidenció la preferencia de los animales por los ensilajes adicionados con tusa, los cuales fueron superiores a los demás con valores de consumos por encima de $6 \mathrm{~kg} /$ día. ARAUJO (2005) estudió los factores que afectan el consumo voluntario de los bovinos encontrando que las características del alimento modifican la conducta alimentaria de los animales, por ello un plan de alimentación debe considerar parámetros de aceptabilidad de los alimentos o suplementos antes de tomar determinaciones finales en cuanto su uso. 
El tiempo dedicado a consumir ensilaje de la parte aérea de la yuca fue relativamente bajo (173 minutos), lo que es diferente a lo reportado en animales en pastoreo por GALLI et al (1996), que aseguran que un bovino pastorea entre 7 y 11 horas por día y que este tiempo de pastoreo depende de factores inherentes a la pastura, al animal y al ambiente, al respecto PENNING et al (1998) estudio el comportamiento ingestivo en bovinos y estableció que vacas secas pastoreaban $451 \mathrm{~min} / \mathrm{día}$, estos valores son diferentes y esa diferencia se atribuye al confinamiento de los animales y a la disponibilidad permanente de los tratamientos, por lo se asume que les tomó menos tiempo para llenar sus requerimientos, en parte también por el mayor contenido de MS del ensilaje en relación al forraje verde. El consumo de MS por parte de los animales fue bajo, este no sobrepaso el $2 \%$ del peso vivo, este bajo consumo pudo presentarse por el manejo realizado, sin embargo, luego de una adecuada adaptación y de una mejor estrategia de suministro, se podrían lograr consumos mayores que permitan que el ensilaje de la parte aérea de la yuca pueda ser incluido en mayores proporciones o como dieta base durante periodos de extrema escases de pasturas. CORRALES y RAMIRES (2008) evaluaron el efecto de la suplementación con ensilaje de maíz y ensilaje de forraje de yuca sobre el desempeño productivo y económico de terneros de levante en el departamento de Sucre, suplementaron a razón del 1,25\% de estos dos ensilajes, obteniendo para el ensilaje de maíz un consumo de $1,3 \mathrm{Kg}$ de MS y para el ensilaje de hoja de yuca un consumo de MS igual a $1,7 \mathrm{Kg}$, vemos el mayor aporte de MS que hace el ensilaje de hoja de yuca y también la aceptación de los animales a este tipo de suplemento, además los autores consiguieron con esta suplementación que los terneros ganaran $539 \mathrm{~g}$ por día, lo que también repercutió en la rentabilidad del sistema.

Las correlaciones entre las variables de calidad y la preferencia (adoptada principalmente por el consumo) resultaron involucrando significativamente las siguientes variables: $\mathrm{pH}$, FDA (fibra detergente acida), HEMIC (hemicelulosa), EE (extracto etéreo) y DIGMS (digestibilidad de la materia seca), esto deja ver que el consumo medio alcanzado por los animales está más relacionado con la misma apetecibilidad o aceptación de los ensilajes adicionados con aditivos que con el consumo potencial del animal y que la principal explicación del mayor consumo obtenido por los tratamientos adicionados con tusa es el pH más bajo que presentaron estos ensilajes, lo cual fue un estímulo o un atrayente a los animales, que desencadeno una mayor tasa de consumo en relación a los demás tratamientos.

Otro aspecto a destacar de los resultados globales de este proyecto de investigación, es la practicidad y la capacidad de general un impacto real en la comunidad de productores y principalmente en los pequeños productores. De esta forma se presenta una solución viable al problema de humedad del ensilaje de la parte aérea de la yuca, al agregar tusa, un material considerado como residuo y que en la actualidad no se le da un manejo adecuado, mejorando así las características de este ensilaje y promoviendo un producto con una mayor cantidad de materia seca y con un importante aporte de nutrientes (alrededor de un $15 \%$ de PB), ideal para suplir la faltante de materia seca en los potreros ocasionada por las variaciones de las lluvias en el trópico bajo.

\section{Conclusiones}

Los ensilajes de la parte aérea de la yuca adicionados con tusa fueron mayormente aceptados por los animales, reflejado esto en mayor número de visitas, mayor tiempo de presencia en el comedero y un mayor consumo. Esta adición promueve un adecuado contenido de materia seca y un importante aporte de nutrientes que podrían mejorar el desempeño animal, además de la posibilidad de usar un recurso descartado. De este modo se destaca la viabilidad de la utilización de este suplemento en los sistemas vacunos de la región caribe cuando la oferta forrajera se ve disminuida por las variaciones de las precipitaciones.

Variables de calidad del ensilaje de la parte aérea de la yuca como $\mathrm{pH}$, hemicelulosa, fibra detergente ácida, extracto etéreo y digestibilidad de la materia seca están correlacionadas con la aceptabilidad por parte de los animales. Principalmente el pH, el cual explica la preferencia por parte de los animales de los ensilajes adicionados con tusa, siendo que estos fueron menores en relación a los demás tratamientos.

\section{Referencias}

AGUILERA, M. 2005. Documento de trabajo sobre economía regional. La economía en el departamento de Sucre: ganadería y sector público. Sincelejo: Banco de la República p.3-10.

ALLINSON, C. 1985. Factors affecting forage intake by range ruminants: a review. Range Manage [serial online]; $1985 \mathrm{~J} .38: 305$. Disponible en: URL: https://journals.uair.arizona.edu/index.php/jrm/article/download/7864/7476 
ARAUJO, O. 2005. Factores que afectan el consumo voluntario en bovinos a pastoreo en condiciones tropicales. IX seminario de pastos y forrales, Universidad de Zulia. Disponible en: URL: http://www.ucv.ve/fileadmin/user upload/facultad agronomia/ Consumo a pastoreo II.pdf

BARAHONA, R; SANCHEZ, S. Limitaciones físicas y químicas de la digestibilidad de pastos tropicales y estrategias para aumentarla. 2005. REVISTA CORPOICA. [serial online]. VOL 6 N¹. Disponible en: URL: http://www.corpoica.org.co/sitioweb/ Revistas/verarticulo.asp?id contenido $=148$

CARVALHO, P; TRINDADE, J; MACARY, S; FISCHER, V; POLI, C; LANG, C. 2007. Consumo de forragens por bovinos em pastejo. Simpósio sobre manejo da pastagens. FEALQ, p 177.

CORRALES, J; RAMIREZ, R. 2008. Efecto de la suplementación con ensilaje de maíz y ensilaje de forraje de yuca en el desempeño productivo y económico de terneros de levante durante la época seca en la subregión sabanas del departamento de sucre. Universidad de Sucre.

CUADRADO, H; MEJÍA, S; CONTRERAS, A; ROMERO, A; GARCÍA, J. Manejo agronómico de algunos cultivos forrajeros y técnicas para su conservación en la región Caribe colombiana. Manual técnico. 2003. Centro de Investigación Turipaná, Corpoica, Cereté (Córdoba, Colombia) [serial online]52p. Disponible en: URL: http://www.agronet.gov.co/www/docs si2/20061024161842 Manejo\%20agronomico\%20forrajeras\%20conservacion.pdf

DI RENZO, J; CASANOVES, F; BALZARINI, M; GONZÁLEZ, L; TABLADO, M; ROBLEDO, C. 2011. InfoStat versión 2011 e. Grupo InfoStat, FCA Universidad Nacional de Córdoba, Argentina. [serial online]. Disponible en: URL: http://www.infostat.com.ar

FERNANDEZ, A. 1999. El silaje y los procesos fermentativos. Silaje de planta entera, Cap. I:4-11. EEA INTA Bordenave.

FERNANDES, DE SOUZAA; OLIVEIRA, R; SANTOS, J; SAYURI M; DA SILVA, A; MATHIAS, H; PERON, P; RODRIGUES, J; DE CARVALHO, D. 2011. Características fermentativas da silagem de híbridos de sorgo sob doses de nitrogênio. Ci. Anim. Bras., Goiânia, [serial online]. v.12, n.3, p. 383-391 Disponible en: URL: http://www.revistas.ufg.br/index.php/vet/article/view/540

FERREIRA, R. A. 2005. Maior produção com melhor ambiente para Aves, Suínos e Bovinos. 1.ed; v.1, Editora Aprenda Fácil, Viçosa/MG. $371 \mathrm{p}$.

GALLI, J; CANGIANO, C; FERNÁNDEZ, H. Comportamiento ingestivo y consumo de bovinos en pastoreo. 1996. Rev. Arg Prod. Anim., [serial online].16(2):119-42. Disponible en: URL: http://www.produccion-animal.com.ar/informacion tecnica/manejo del alimento/15-ingestivo y consumo bovinos.pdf

GARCÉS, AM; BERRIO, L; RUIZ S; SERNA, J; BUILES, A. Ensilaje como fuente de alimentación para el ganado. 2004. Revista Lasallista de Investigación. [serial online]. Vol 1(1): 66-71 Disponible en: URL: http://www.lasallista.edu.co/fxcul/media/pdf/Revista/ Vol1n1/066-71\%20Ensilaje\%20como\%20fuente\%20de\%20alimentaci\%C3\%B3n\%20para\%20el\%20ganado.pdf

MARTÍNEZ, E; PULIDOR; LATRILLE, L. Efecto de la paja de trigo tratada con álcali sobre el consumo de alimento y comportamiento ingestivo de vacas lecheras. 2002. Archivos de medicina veterinária. [serial online]. 34:1-14 Disponible en: URL: http://www.scielo. cl/scielo.php?pid=S0301-732X2002000200006\&script=sci arttext

MEDRANO, J. Factores que influencian el consumo voluntario de rumiantes en pastoreo. 1991. Programa regional de investigación pecuaria, CORPOICA[serial online]. Disponible en: URL: http://corpomail.corpoica.org.co/BACFILES/BACDIGITAL/20527/20527. $\underline{\text { pdf }}$

ORTEGA, L; HUCHIMA, C; RIVAS, F. A. Conducta ingestiva de bovinos Cebú adultos en leucaena manejada a dos alturas diferentes. 2009. Técnica Pecuaria Méxicana [serial online]. 47(2):125-134. Disponible en: URL: http://www.tecnicapecuaria.org. mx/trabajos/200904023416.pdf

PATIÑO, R; GONZÁLEZ, K; PORRAS, F; SALAZAR, L; VILLALBA, C; GIL, J. 2008. Comportamiento ingestivo diurno y desempeño de novillos en pastoreo pertenecientes a tres grupos genéticos durante dos épocas climáticas. Disponible en: URL: http://www. Irrd.org//rrd20/3/pati20036.htm 
PENNING, P; PARSONS, A; NEWMAN, J; ORR R; HARVEY, A. Behavioural and physiological factors limiting intake in grazing ruminants. 1998. Pasture ecology and animal intake [serial online]. p. 10-20. Disponible en: URL: http://www1.foragebeef. ca/\$Foragebeef/frgebeef.nsf/all/frg743/\$FILE/grazingbehaviourruminants.pdf

ROCERO, D. 2002. Evaluacion, producción y calidad del forraje de yuca Manihot esculenta Crantz con corte periódico manual. Universidad Nacional de Colombia, Facultad de ciencias agropecuarias.

SÁNCHEZ, L. Estrategias modernas para la conservación de forrajes en sistemas de producción bovina tropical. 2005. Revista Corpoica [serial online]. 6(2):69-81. Disponible en: URL: http://www.corpoica.org.co/sitioweb/Archivos/Revista/8 EstrategiasMod pp.PDF 